\title{
Adaptive Modulation Coding Method based on Minimum Packet Loss Rate in AOS Communication System
}

\author{
Qingli Liu*, Yanjun Yang, and Zhiguo Liu \\ National and Local Joint Engineering Laboratory of Intelligent Shipping and Logistics Network Technology, Dalian University, Dalian, 116622, China
}

\begin{abstract}
Aiming at the problem of increasing packet loss rate and decreasing throughput caused by the characteristics of high data burst and high channel error rate in the AOS space communication system, this paper considers the factors of queue packet loss and transmission error packet loss and proposes an adaptive modulation coding method based on minimum packet loss rate in the AOS communication system. Firstly, a system packet loss objective function is established, and the modulation coding mode can be determined by solving the objective function minimum. Secondly, the modulation coding mode is dynamically adjusted according to certain rules, determined by the channel state and the queue state jointly. Finally, the system packet loss rate is reduced and the transmission performance of the system is improved. The theoretical analysis and simulation results show that compared with the SLBCCQ method, this method can reduce the system packet loss rate by up to $30 \%$. Meanwhile, compared with the AMC algorithm, it can reduce the system packet loss rate by $41.7 \%$.
\end{abstract}

Keywords: AOS; adaptive modulation coding; packet loss rate

(Submitted on November 16, 2018; Revised on December 15, 2018; Accepted on January 11, 2019)

(C) 2019 Totem Publisher, Inc. All rights reserved.

\section{Introduction}

With the rapid development of the technology of aerospace, manned spacecraft, and orbital space stations, human space activities have entered the era of space stations, and complex space missions have put forward many difficult data processing requirements [1-3]. Advanced Orbiting System (AOS) is a data transmission protocol suitable for satellite communication networks, and it was designed to solve the transmission problem of complex service type data in current satellite links and the diverse and complex space task demands [4]. AOS is a new generation of satellite communication network protocol proposed by the Spatial Data Advisory Committee to solve the transmission problem of traffic streams with high-rate and strong-burst characteristics in space links [5]. In order to satisfy the complex requirements of data processing and transmission, such as multi-user, high-capacity, high-rate, and bi-directional transmission, the error detection and error correction can be used for reliability and accuracy of data transmission [6].

AOS has the characteristics of large data transmission, high burst, high error rate, and time-varying channel fading. These features put forward new requirements for the reliability and transmission efficiency of space communication [7-9]. In the research on the reliability and transmission efficiency of space communication, reference [10] applied Type-II and Type-III Hybrid Automatic Retransmission Request (HARQ) to wireless communication systems and used the cross-layer design method of the physical layer and data link layer to reduce the packet loss rate and improve spectrum utilization. Reference [11] proposed a prediction-based HARQ algorithm, which achieved good results in bit error rate, transmission times, and transmission efficiency. Reference [12] combined adaptive modulation coding technology and channel interleaving technology to better adapt to time-varying fading channels, effectively improve system throughput, and significantly enhance system BER performance. The above documents have improved the system performance by studying reliability and transmission efficiency, but they do not take into account the characteristics of random arrival data stream and high data burst.

\footnotetext{
* Corresponding author.

E-mail address: lq10808@ sina.com
} 
Aiming at the characteristics of random arrival data stream and high data burst, reference [13] considered finite length queue and adaptive modulation coding and proposed an Adaptive Modulation Coding (AMC) algorithm to improve the average throughput of the system. However, the algorithm does not consider the overflows in a queue with a limited length, when the number of data packets is increasing in a certain period of time. This will result in an increase in the number of lost packets. Reference [14] proposed a scheme based on the low buffer at the data link layer and the choice of channel quality (SLBCCQ). Although the average throughput performance was improved, it does not obtain the optimal transmission performance, because of the series of modulation and coding modes according to the channel state.

Therefore, an adaptive modulation coding method based on minimum packet loss rate (AMCMMPLR) in the AOS space communication system is proposed in this paper. It first establishes the system packet loss objective function and determines the modulation coding mode by solving the objective function minimum. Then, it dynamically adjusts the modulation coding mode according to certain rules, which are determined by the channel state and the queue state. When the number of data packets overflowing from the queue is large, the modulation coding mode is upgraded to ensure that there are fewer packets lost in the queue. The queue packet loss rate and ultimately the system packet loss rate are reduced. When the queue is idle, the modulation coding mode is downgraded to ensure the reliable transmission of data. Finally, the transmission error packet loss rate and the system packet loss rate are reduced.

\section{System Model}

Figure 1 shows the flow chart of the system module. The data link layer at the transmission end has a packet buffer queue, and the physical layer has a modulation and coding mode controller and a signal transmitter. The physical layer of the receiving end includes the channel estimation module, predictive modulation and coding mode module, modulation and coding mode adjustment module, modulation and coding mode selector, and receiver.

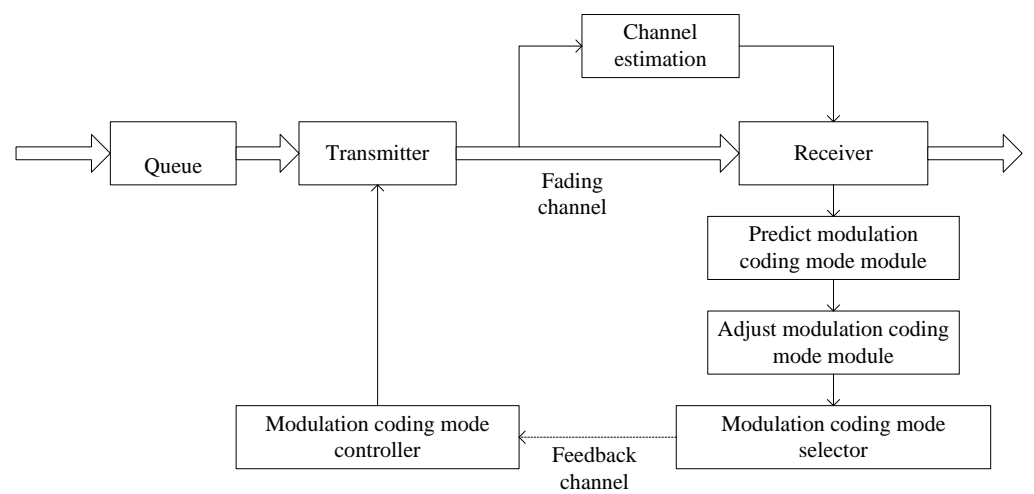

Figure 1. System block diagram based on adaptive modulation coding design

The transmitter uses a finite length queue (buffer) by First in First out (FIFO). When data packets at the data link layer arrive, they are queued in the buffer. The system determines the selected modulation and coding scheme (MCS) according to the feedback information from the receiver. The number of data packets that should be sent in the current time slot is determined according to different modulation and coding modes. At the receiving end, the channel state is obtained through channel estimation, and the queue state is obtained at the same time. According to the minimum packet loss in the system, the modulation coding mode is preliminarily determined, and then the modulation coding mode is dynamically adjusted. Finally, the selected modulation coding mode is saved and fed back to the transmission end.

\section{Establishment of Objection Function}

\subsection{Objective Function}

As can be seen from Figure 1, packet loss in the AOS space communication system is mainly affected by two factors: queue loss and transmission error packet loss. 1) Queue packet loss: packets enter the sender's cache queue before sending and wait for sending. If the queue is full, the packet will be discarded, resulting in packet loss in the queue. 2) Transmission error packet loss: due to channel fading or interference, the receiver cannot decode the received data correctly and will discard the data packet. Therefore, it is necessary to consider the effects of queue packet loss and transmission error packet loss, reduce system packet loss, improve average throughput, and optimize the performance of the AOS communication system.

When the data packet is not discarded by the queue and is correctly accepted through the fading channel, it indicates 
that the data packet is correctly accepted. During the $t$ period, the queue overflows $D_{t}$ packets, and the transmission error packets are $S_{t}$. If the loss packets of system are least in the t period, it will make the $D_{t}+S_{t}$ least and $\left|D_{t}-S_{t}\right|$ least. Therefore, the objective function $\xi$ is expressed as Equation (1).

$$
\zeta=\left|D_{t}-S_{t}\right| \times \exp \left(D_{t}+S_{t}\right)
$$

\subsection{Calculation of Transmission Error Packets}

The adaptive modulation and coding selects an appropriate modulation and coding mode according to the state of the channel, in which a specified packet error rate is satisfied, and maximizes the data transmission rate. It is assumed that the data is transmitted at a constant power and the entire signal-to-noise ratio (SNR) range is divided into $N$ consecutive nonoverlapping sub-intervals. The channel is divided into $N$ finite states. The division of the channel state determines the threshold value of the adjacent state interval. Let $\left\{\gamma_{n}\right\}_{n=0}^{n=N+1}$ denote the threshold value of the adjacent state. When $\gamma \in$ $\left[\gamma_{n}, \gamma_{n+1}\right)$ is satisfied, the channel is in $n$ state. This paper uses the rules of $\overline{P E R_{n}}=P_{0}$ to divide the channel state. The specific steps are as follows.

- Step 1. Set $n=N$ and $\gamma_{N+1}=+\infty$.

- Step 2. For each $n$, search for the unique $\gamma_{n} \in\left[\gamma_{0}, \gamma_{n+1}\right)$ and $\overline{P E R_{n}}=P_{0}$.

- Step 3. If $n>1$, set $n=n-1$, and go to Step 2; otherwise, go to Step 4 .

- Step 4. Set $\gamma_{0}=0$.

SNR $\gamma$ is a random variable whose probability density function is expressed as Equation (2).

$$
p_{\gamma}(\gamma)=\frac{m^{m} \gamma^{m-1}}{\bar{\gamma}^{m} \tau(m)} \exp \left(-\frac{m \gamma}{\bar{\gamma}}\right)
$$

Parameter $m$ is the channel fading parameter $\left(m \geq \frac{1}{2}\right)$. When $m=1$, it indicates the Rayleigh channel [15]. $\bar{\gamma}$ is the average SNR of the channel. $\tau(m)=\int_{0}^{\infty} t^{m-1} \exp (-t) \mathrm{d} t$ is a gamma function.

In this paper, five effective modulation and coding modes are selected. Mode 0 indicates that the system does not transmit information. The modulation coding mode and error estimation parameters used are shown in Table 1.

Table 1. Modulation coding mode and error estimation parameters

\begin{tabular}{|c|c|c|c|c|c|}
\hline Modulation coding mode & 1 & 2 & 3 & 4 & 5 \\
\hline Modulation & BPSK & QPSK & QPSK & 16-QAM & 64-QAM \\
\hline Coding rate & $1 / 2$ & $1 / 2$ & $3 / 4$ & $3 / 4$ & $3 / 4$ \\
\hline Transmission rate & 0.5 & 1 & 1.5 & 3 & 4.5 \\
\hline$a_{k}$ & 247.7229 & 90.2514 & 67.6181 & 53.3987 & 35.3508 \\
\hline$g_{k}$ & 7.9932 & 3.4998 & 1.6883 & 0.3756 & 0.0900 \\
\hline$\gamma_{p k}(\mathrm{~dB})$ & -1.5331 & 1.0942 & 3.9772 & 10.2488 & 15.9784 \\
\hline$\gamma_{p k}$ & 0.706 & 1.2865 & 2.495 & 10.5896 & 396132 \\
\hline
\end{tabular}

For simplicity, the packet error rate curve under the influence of white Gaussian noise is fitted, as shown in Equation (3).

$$
\operatorname{PER}_{k}(\gamma) \approx \begin{cases}1, & \text { if } 0<\gamma<\gamma_{p k} \\ a_{k} \exp \left(-g_{k} \gamma\right), & \text { if } \gamma \geq \gamma_{p k}\end{cases}
$$

Where $k$ represents a different modulation and coding mode. $\gamma_{p k}, g_{k}$, and $a_{k}$ are constants associated with mode $k$.

The probability of channel state $n$ that can be selected is shown as Equation (4).

$$
\operatorname{Pr}(n)=\int_{\gamma_{n}}^{\gamma_{n+1}} p_{\gamma}(\gamma) \mathrm{d} \gamma=\frac{\tau\left(m, \frac{m \gamma_{n}}{\bar{\gamma}}\right)-\tau\left(m, \frac{m \gamma_{n+1}}{\bar{\gamma}}\right)}{\tau(m)}
$$


$\tau(m, x)=\int_{x}^{\infty} t^{m-1} \exp (-t) \mathrm{d} t \cdot \gamma_{n}$ and $\gamma_{n+1}$ are respectively the lower and upper boundary points of the SNR interval when the channel state is $n$.

Then, the average error rate $\overline{P E R_{n, k}}$ of the $n$ state using the modulation coding mode $k$ is expressed as Equation (5).

$$
\begin{aligned}
\overline{P E R_{n, k}} & =\frac{1}{\operatorname{Pr}(n)} \int_{\gamma_{n}}^{\gamma_{n+1}} a_{k} \exp \left(-g_{k} \gamma\right) p_{\gamma}(\gamma) \mathrm{d} \gamma \\
& =\frac{1}{\operatorname{Pr}(n)} \frac{a_{k}}{\tau(m)}\left(\frac{m}{\gamma}\right)^{m} \frac{\tau\left(m, b_{k} \gamma_{n}\right)-\tau\left(m, b_{k} \gamma_{n+1}\right)}{\left(b_{k}\right)^{m}}, \quad n=1, \cdots, N
\end{aligned}
$$

The parameter $b_{k}$ is expressed as Equation (6).

$$
b_{k}=m / \bar{\gamma}+g_{k}
$$

The transmission error rate per in the t period is expressed as Equation (7).

$$
\operatorname{per}= \begin{cases}\frac{\int_{\gamma_{n}}^{\gamma_{n+1}} a_{k} \exp \left(-g_{k} \gamma\right) p_{\gamma}(\gamma) \mathrm{d} \gamma}{\operatorname{Pr}(n)}, & \text { if } \gamma_{p k}<\gamma_{n} \\ 1, & \text { if } \gamma_{p k}>\gamma_{n+1} \\ \frac{\operatorname{Pr}\left(\gamma_{n}, \gamma_{p k}\right)}{\operatorname{Pr}(n)}+\frac{\int_{p k}^{\gamma_{n+1}} a_{k} \exp \left(-g_{k} \gamma\right) p_{\gamma}(\gamma) \mathrm{d} \gamma}{\operatorname{Pr}\left(\gamma_{n}, \gamma_{p k}\right)}, & \text { if } \gamma_{n}<\gamma_{p k}<\gamma_{n+1}\end{cases}
$$

The transmission error packet $S_{t}$ in the t period is shown as Equation (8).

$$
S_{t}=\min \left(U_{t-1}, C_{k}\right) \times p e r \times H
$$

Where $U_{t-1}$ is the number of packets in the queue at the beginning of the $t$ period.

$H$ represents the adjustment factor and is expressed as Equation (9).

$$
H=\frac{C_{a v g}}{\lambda T_{f}}
$$

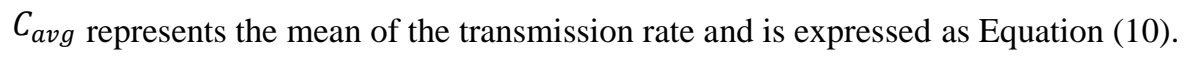

$$
C_{\text {avg }}=\sum_{k=1}^{5} C_{k} \operatorname{Pr}(k)
$$

\subsection{Calculation of Queue Packet Loss}

At the beginning of t unit time, there are $U_{t-1}$ packets in the queue. At this time of the channel state, the transmitter can send $C_{t}$ packets. When the time tends, the number of packets arriving in the period is $A_{t}$. The $C_{t}$ packets sent from the queue will not be deleted from the queue immediately but will wait for the feedback information from the receiver. When adding the incoming $A_{t}$ packets, there are $U_{t}$ packets in the queue. We will analyze the arrival process, queue service process, and queue state recursion of packets respectively and construct a finite state Markov chain. Queue packet loss is related to the queue length, the arrival of the packet, and the selection of the modulation code. 


\subsubsection{Arrival Process}

$t$ represents the time unit. $A_{t}$ represents the number of packets arriving at the t period. The arrival process $A_{t}$ is independent of the queue status and channel state. We assume that $A_{t}$ is Poisson distributed with parameter $\lambda T_{f}$. The expectation of $A_{t}$ satisfies $\mathrm{E}\left\{A_{t}\right\}=\lambda T_{f}$. The probability of the number of unit time packets arriving is a, which is expressed as Equation (11).

$$
\operatorname{Pr}\left(A_{t}=a\right)=\left\{\begin{array}{l}
\frac{\left(\lambda T_{f}\right)^{a} \exp \left(-\lambda T_{f}\right)}{a !}, \text { if } \mathrm{a} \geq 0 \\
0, \quad \text { otherwise }
\end{array}\right.
$$

The range of $A_{t}$ can be expressed as Equation (12).

$$
A_{t} \in A:=\{0,1, \cdots, \infty\}
$$

\subsubsection{Queue Service Process}

Adaptive modulation coding is different from non-adaptive modulation coding. It is a dynamic service process, and the data packets transmitted in unit time are variable. $C_{t}$ represents the number of packets transmitted during the $\mathrm{t}$ period. For each transmission mode $k$, let $C_{k}$ denote the number of packets transmitted per time period. The range of $C_{t}$ is expressed as Equation (13).

$$
C_{t} \in C, C:=\left\{C_{0}, C_{1}, \cdots, C_{K}\right\}
$$

$C_{k}$ takes a positive integer. If the code rate of the modulation coding mode is 1 and per frame can accommodate b packets, $C_{k}$ is expressed as Equation (14).

$$
C_{k}=b R_{k}
$$

\subsubsection{Queue State Recursion}

The queue state recursion model is shown in Figure 2.

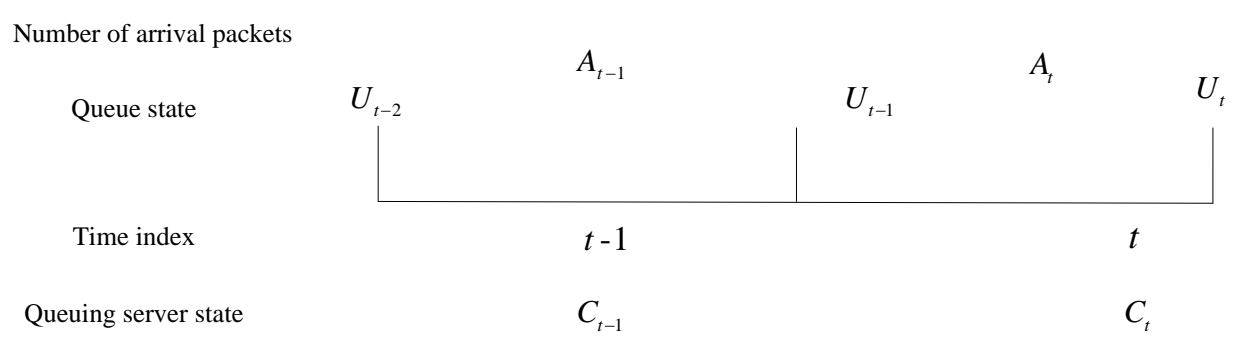

Figure 2. Queue state recursion model

The model described in Figure 2 is used to describe the queue state. $A_{t}$ represents the number of packets arriving within the $t$ time period. $U_{t}$ represents the state of the queue (the number of packets in the queue) at the end of the $t$ period. $C_{t}$ represents the number of packets transmitted in the $t$ time period.

Assuming the size of the queue cache is $Q$, it can accommodate up to $Q$ packets at most. The set of $\mathrm{u}$ is expressed as Equation (15).

$$
U_{t} \in u:=\{0,1, \cdots, Q\}
$$

It assumes that the packet is first removed from the queue at the beginning of time $t$, based on the service state $C_{t}$. The arriving packets are placed in the queue for the $t$ period. When $C_{t}$ packets are removed from the queue, the number of 
packets left in the queue is expressed as Equation (16).

$$
L_{t}=\max \left\{0, U_{t-1}-C_{t}\right\}
$$

The size of the free space of the queue at the beginning of time $t$ can be expressed as Equation (17).

$$
F_{t}=Q-L_{t}=Q-\max \left\{0, U_{t-1}-C_{t}\right\}
$$

Now, the packets arriving need to be considered in the $t$ period. If $A_{t} \leq F_{t}$, all arriving packets enter the queue and the queue status is $U_{t}=L_{t}+A_{t}$. If $A_{t}>F_{t}$, only $F_{t}$ packets enter the queue and $A_{t}-F_{t}$ packets will be discarded, and the queue status is $U_{t}=Q$. Therefore, the queue status is expressed as Equation (18).

$$
U_{t}=\min \left\{Q, \max \left\{U_{t-1}-C_{t}\right\}+A_{t}\right\}
$$

$D_{t}$ represents the number of packets discarded during the t period, and $D_{t}$ is related to $U_{t-1}, A_{t}$, and $C_{t}$. The expression of $D_{t}$ is shown as Equation (19).

$$
D_{t}=\max \left\{0, A_{t}-Q+\max \left\{0, U_{t-1}-C_{t}\right\}\right\}
$$

\section{Adaptive Modulation Coding Method based on AOS Communication System with Minimum Packet Loss Rate}

It is assumed that the Doppler frequency shift $f_{d}$, the average received SNR $\bar{\gamma}$, the channel fading parameter $m$, the fixed resource allocation parameter $b$, the receiving end queue length $Q$, and the unit time packet arrival rate $\lambda T_{f}$ are known. The specific steps of the adaptive modulation coding method based on the minimum packet loss rate of the AOS communication system are as follows.

- Step 1. Judge whether the queue cache is empty.

- Step 2. If the queue cache is non-empty, predict the modulation coding mode and adjust the modulation coding mode. Then, skip to step 3; otherwise, skip to step 6.

- Step 3. Transmit data.

- Step 4. Judge whether the data transmission is wrong.

- Step 5. If the data transmission error occurs, update the packet loss rate data and skip to step 6; otherwise, skip to step 6.

- Step 6. Packet arrival.

- Step 7. Judge whether the queue overflows.

- Step 8. If the queue does not overflow, skip to step 9; otherwise, update the packet loss rate data and skip to step 1.

- Step 9. Judge whether the data transmission is completed.

- Step 10. If the data transmission is complete, skip to step 11; otherwise, skip to step 1.

- Step 11. End.

The flow chart of modulation coding distribution is shown in Figure 3.

\section{Dynamically Adjust the Modulation Coding Mode}

Assume that the Doppler frequency shift is $f_{d}=10 \mathrm{HZ}$, the channel fading parameter is $m=1$, the average accepted SNR is $\bar{\gamma}=20 \mathrm{~dB}$, the system allocation parameter is $b=2$, the queue length is $Q=20$, and the unit time packet arrival rate is $\lambda T_{f}=2$. The number of channel states is 6 . The channel states are sorted from small to large. A higher channel state represents a better channel quality.

As shown in Table 2, the modulation coding mode is determined under the premise that the objective function is the minimum. In the table, if the channel state is mode 0 , the modulation coding mode is determined by considering the queue state alone, and the modulation coding mode 0 is selected to not transmit data. When the number of packets in queue is large, the finite length queue will overflow, and the queue packet loss will be significantly increased. This will result in the system packet loss increases. It can be seen that determining the modulation coding mode by channel state alone is not optimal. 
When the queue cache has the possibility of overflow, the modulation coding mode is increased by one level based on the channel state. In other cases, the modulation coding mode is determined by the channel state. In the table, when the channel state is mode 3 and the remaining two data packets are in the queue, the modulation coding mode 2 is sufficient to transmit the data packet buffered by the queue. It is seen that this scheme is also not optimal. However, if only the objective function is guaranteed to be the least, there are some problems. When the channel status is mode 0 and the queue buffer is large, the packets transmitted in the modulation coding mode 2 are discarded. This does not reduce system packet loss. When the channel status is 2 and there are two packets in the queue, the modulation coding mode 1 is selected. Although the transmission error packet will be reduced, it will increase the pressure of the queue and cause the queue to drop packets. Therefore, the selected modulation coding mode needs to be modified. If the level of the channel state is to be degraded, the degraded modulation coding mode must be sufficient to transmit the data of the queue buffer. If the level of the channel state is to be upgraded, the transmission error rate of the upgraded modulation coding mode is less than one. The adjusted modulation and coding modes are shown in Table 3.

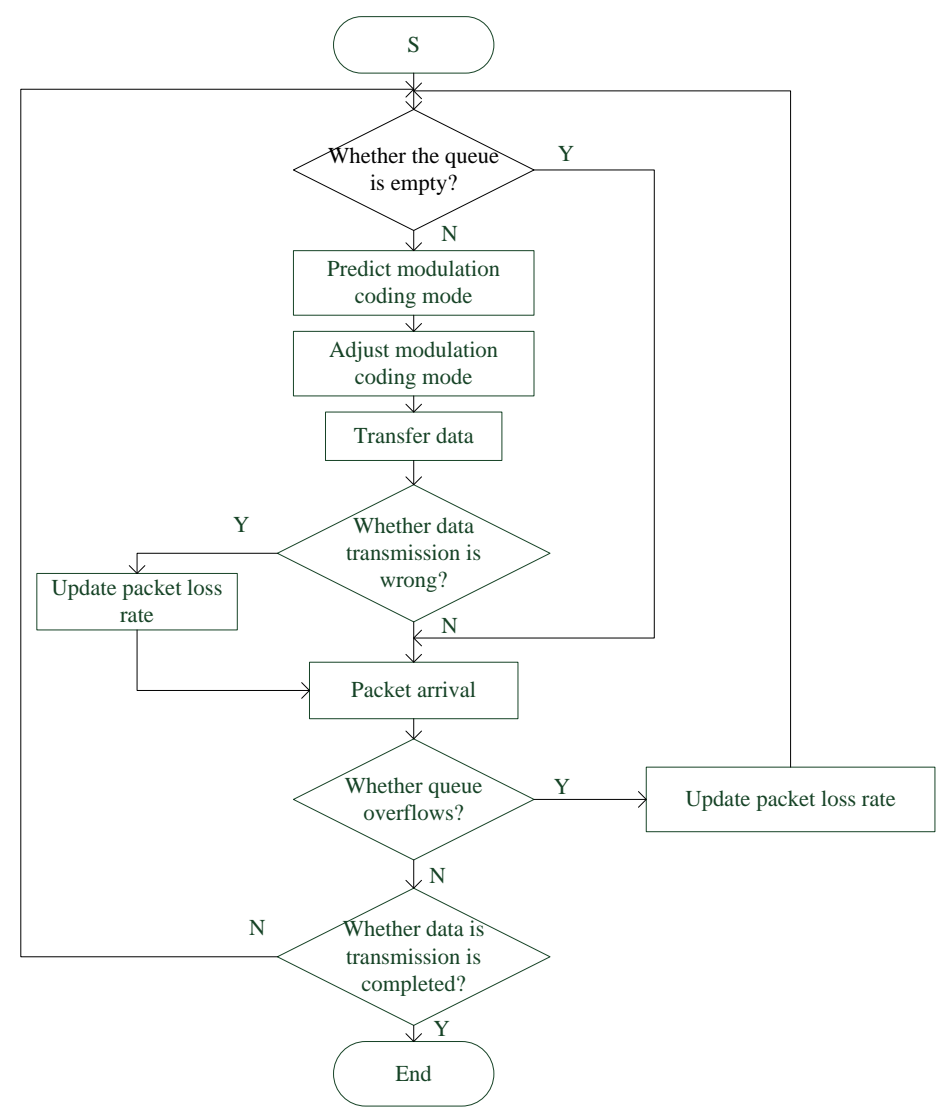

Figure 3. Modulation coding distribution flow chart

Table 2. Modulation coding mode table

\begin{tabular}{|c|c|c|c|c|c|c|}
\hline $\begin{array}{l}\text { The number } \\
\text { of remaining } \\
\text { packets in the queue }\end{array}$ & 0 & 1 & 2 & 3 & 4 & 5 \\
\hline 0 & & & & & \\
\hline 1 & 0 & 0 & 0 & 0 & 0 & 0 \\
\hline 2 & 0 & 0 & 1 & 1 & 1 & 1 \\
\hline 3 & 0 & 1 & 1 & 2 & 2 & 2 \\
\hline 4 & 0 & 1 & 1 & 2 & 3 & 3 \\
\hline 5 & 0 & 1 & 2 & 2 & 3 & 4 \\
\hline 6 & 0 & 1 & 2 & 3 & 3 & 4 \\
\hline 7 & 1 & 1 & 2 & 3 & 4 & 4 \\
\hline 8 & 1 & 2 & 2 & 3 & 4 & 4 \\
\hline 9 & 1 & 2 & 3 & 3 & 4 & 5 \\
\hline
\end{tabular}


Table 3. Adjusted modulation coding mode table

\begin{tabular}{|c|c|c|c|c|c|c|}
\hline $\begin{array}{l}\text { The number } \\
\text { of remaining } \\
\text { packets in the queue }\end{array}$ & 0 & 1 & 2 & 3 & 4 & 5 \\
\hline 0 & & & & & \\
\hline 1 & 0 & 0 & 0 & 0 & 0 & 0 \\
\hline 2 & 0 & 1 & 1 & 1 & 1 & 1 \\
\hline 3 & 0 & 1 & 2 & 2 & 2 & 2 \\
\hline 4 & 0 & 1 & 2 & 3 & 3 & 3 \\
\hline 5 & 0 & 1 & 2 & 3 & 4 & 4 \\
\hline 6 & 0 & 1 & 2 & 3 & 4 & 4 \\
\hline 7 & 0 & 1 & 2 & 3 & 4 & 4 \\
\hline 8 & 1 & 1 & 2 & 3 & 4 & 5 \\
\hline 10 & 1 & 2 & 2 & 3 & 4 & 5 \\
\hline
\end{tabular}

\section{Experimental Simulation}

\subsection{Simulation Parameter Setting}

The AMCMMPLR in the AOS space communication system is simulated, and the simulation parameters are shown in Table 4.

Table 4. Adjusted modulation coding mode table

\begin{tabular}{|c|c|}
\hline Simulation parameters & Parameter values \\
\hline Doppler frequency shift $f_{d}$ & $10 \mathrm{HZ}$ \\
\hline Channel fading parameter $m$ & 1 \\
\hline Modulation coding format & BPSK1/2, QPSK1/2,QPSK3/4,16QAM3/4,64QAM3/4 \\
\hline Queue length $Q$ & 20 data packets \\
\hline Average signal-to-noise ratio & $20 \mathrm{~dB}$ \\
\hline Target packet error rate $P_{0}$ & 0.001 \\
\hline Resource allocation parameter $b$ & 2 data packets \\
\hline
\end{tabular}

\subsection{Simulation Results and Analysis}

The numerical results in Figure 4 show that the system transmission error rate of the AMC method does not change with the increase in the Poisson arrival rate. The system transmission error rate of AMCMMPLR and SLBCCQ keeps increasing as the Poisson's arrival rate increases. When the Poisson reach rate increases, the queue pressure will become bigger and the queue loss will increase. The SLBCCQ and AMCMMPLR will select a more advanced modulation and coding mode to reduce the pressure of the queue. Although they reduce the packet loss and sacrifice the reliability of the transmission, the transmission error rate of the system will be increased. The system transmission error rate of AMCMMPLR is basically lower than that of SLBCCQ in Figure 4. The AMCMMPLR scheme considers that the low-level modulation coding mode is sufficient to transmit the data packets in the queue buffer when the data packets in the queue buffer are less.

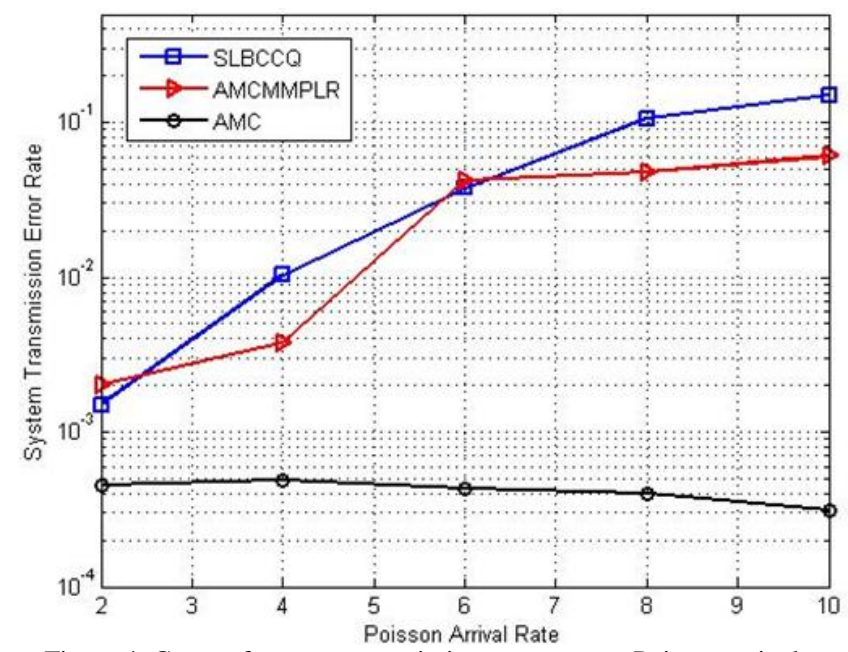

Figure 4. Curve of system transmission error rate on Poisson arrival rate 
The numerical results in Figure 5 show that the system queue loss rate of AMC, SLBCCQ, and AMCMMPLR will increase as the Poisson's arrival rate increases. The system queue loss rate of SLBCCQ and AMCMMPLR is less than that of AMC. When the Poisson arrival rate increases, the queue pressure will become bigger and the queue loss will increase. The SLBCCQ and AMCMMPLR select a more advanced modulation and coding mode to reduce the queue pressure and queue packet loss rate. The system queue loss rate of AMCMMPLR is between SLBCCQ and AMC, because it takes into account the impact of the transmission error rate.

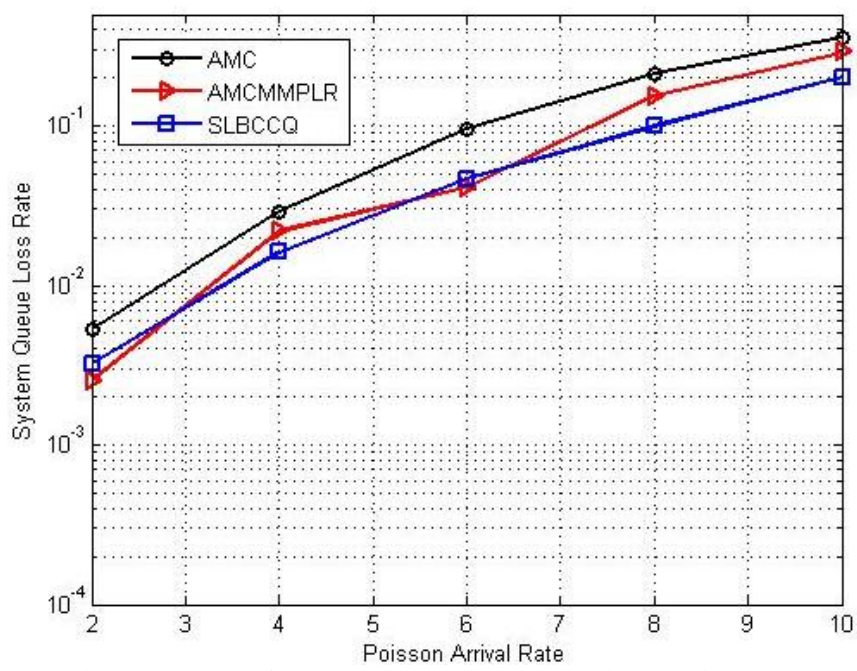

Figure 5. Curve of system packet loss rate on Poisson arrival rate

The numerical results of Figure 6 show that the system package loss rate of AMCMMPLR is lower than that of AMC and SLBCCQ. The AMCMMPLR can reduce the system packet loss rate by up to 30\% compared with the SLBCCQ. Meanwhile compared with the AMC algorithm, it can reduce the system packet loss rate by $41.7 \%$. When the Poisson arrival rate is relatively low, the system packet loss rate of AMCMMPLR is much lower than that of SLBCCQ. The AMCMMPLR will choose a lower-level modulation-coding mode and guarantee that data packets in the queue buffer can be transmitted when the data packets in the queue buffer are less. When the Poisson arrival rate is relatively high, the system packet loss rate of AMC, SLBCCQ, and AMCMMPLR is basically the same. Their system packet loss rate will reach a balance regardless of how the relationship between transmission error and queue loss is adjusted.

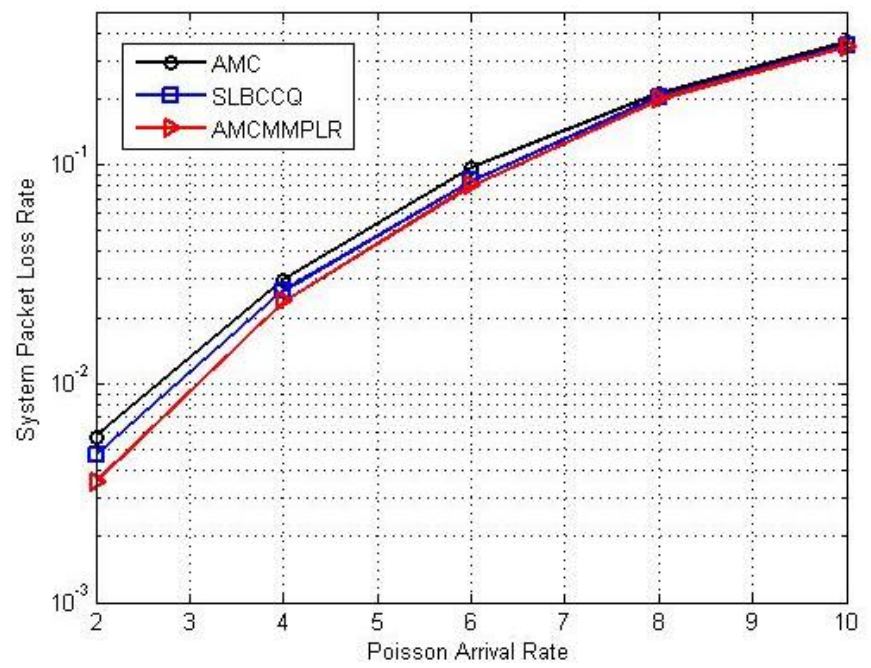

Figure 6. Curve of system packet loss rate on Poisson arrival rate

\section{Conclusions}

Considering the characteristics of AOS space communication, an adaptive modulation coding method based on minimum packet loss rate in the AOS space communication system is proposed in this paper. The method synthesizes the queue state and channel state and establishes the objective function. It will determine the modulation and coding mode by solving the 
minimum of the objective function. It will adjust the modulation and coding mode according to certain rules, considering the balance of channel state and queue state. The simulation results show that the proposed adaptive modulation coding method based on minimum packet loss rate in the AOS space communication system can reduce the system packet loss rate and improve the system performance.

\section{Acknowledgements}

This work was partly financially supported through grants from the National Natural Science Foundation of China (No. 61571074) and the Innovation Support Program for High-level Personnel of Dalian (No. 2016RQ076).

\section{References}

1. Q. L. Liu, C. S. Pan, and G. R. Wang, "Optimized Link Adaptation based on Virtual Modulation and Coding," Journal of System Simulation, Vol. 24, No. 11, pp. 97-101+106, 2012

2. Y. Hao, Z. D. Xiao, and Q. Pan, "Relationship Between Data Frame Length and Throughput of AOS Protocol," Science and Technology Innovation Herald, Vol. 27, pp. 23-23, 2008

3. J. Jiao, Q. Y. Zhang, H. Li, A. G. Li, and P. Chen, "Expected File Delivery Time of Prompt NAK Mode in CCSDS File Delivery Protocol," Journal of Astronautics, Vol. 30, No. 01, pp. 260-265, 2009

4. K. Jeeja and C. Dutta, "Test Station for CCSDS based Data Handling Systems of Mini Satellites," in Proceedings of 2012 IEEE International Conference on Advanced Communication Control and Computing Technologies, pp. 10-13, 2012

5. L. S. Liu, J. C. Hu, and Y. F. Ma, "Research on Queue Management Algorithm for Different Satellite Source Types in AOS," Science \& Technology Information, Vol. 16, No. 12, pp. 10-11, 2018

6. W. X. Lu, "Research of AOS Receiver Distributed Simulation Systems based on HLA-RTI," Shenyang Ligong University, 2017

7. Q. P. Zheng, "Optimal Design on Data Delivery Protocol in Deep Space Communications," Harbin Institute of Technology, 2013

8. Z. J. Song, C. M. Wang, and M. Zhang, "Analysis and Research of SCPS-TP in Satellite Communication Network," Electronic Design Engineering, 2018

9. Z. Wang and Y. Dong, "The Remote Virtual-Channel Transfer Protocol," in Proceedings of Spacewire Conference IEEE, pp. 14, 2014

10. C. L. Mang, "Research of the Adaptive Modulation and Coding and Automatic Repeat Request based on Advanced Orbiting," Shenyang Ligong University, 2013

11. Q. Zhang, "The Research of Hybrid Automatic Request Algorithm based on Packet Loss Prediction," University of Electronic Science and Technology of China, 2016

12. C. Q. Li, "Research on Key Technologies and Applications of Adaptive Transmission," Shangdong University, 2011

13. Q. Liu, S. Zhou, and G. B. Giannakis, "Queuing with Adaptive Modulation and Coding over Wireless Links: Cross-Layer Analysis and Design," IEEE Press, 2005

14. N. Xiong, "Research on Link Adaptation Schemes based on Cross-Layer Design," Huazhong University of Science and Technology, 2013

15. D. Luengo and L. Martino, “Almost Rejectionless Sampling from Nakagami-M Distributions,” Electronics Letters, Vol. 48, No. 24, pp. 1559-1561, 2012

Qingli Liu received his Ph.D. from Northeast University in 2012. Since 2014, he has been an associate professor in the Information Engineering College at Dalian University. He was a visiting scholar in the Department of Electronic Engineering at Utah State University from September 2015 to March 2016. His research interests include satellite network protocol, network architecture and design, and network technologies.

Yanjun Yang received her bachelor's degree from Lingnan Normal University in 2016. Since September 2016, she has been a master's student at Dalian University. Her main research area is satellite networks.

Zhiguo Liu studied in the Information Science and Engineering College at Northeast University and received his M.S. degree and Ph.D. in 2000 and 2003, respectively. He is currently a professor in the Information Engineering College at Dalian University. His main research areas are satellite network protocol, network architecture and design, and network technologies. 